\title{
CLEANING TECHNIQUES FOR APPLIED-B ION DIODES
}

\author{
M. E. Cuneo, P. R. Menge, D. L. Hanson, W. E. Fowler, M. A. Bernard, G. R. Ziska, \\ A. B. Filuk, J. E. Bailey, M. P. Desjarlais, T. R. Lockner, T. J. Nash, D. D. Noack, S. A. Slutz \\ Sandia National Laboratories, Albuquerque, NM 87185-1193. \\ D. R. Welch \\ Mission Research Corporation, Albuquerque, NM 87106.
}

\begin{abstract}
Measurements and theoretical considerations indicate that the lithium-fluoride ( $\mathrm{LiF}$ ) lithium ion source operates by electron-assisted field-desorption, and provides a pure lithium beam for 10-20 ns. Evidence on both the SABRE (1 TW) and PBFA-II (20 TW) accelerators indicates that the lithium beam is replaced by a beam of protons, and carbon resulting from electron thermal desorption of hydrocarbon surface and bulk contamination with subsequent avalanche ionization. Appearance of contaminant ions in the beam is accompanied by rapid impedance collapse, possibly resulting from loss of magnetic insulation in the rapidly expanding and ionizing neutral layer. Electrode surface and source substrate cleaning techniques are being developed on the SABRE accelerator to reduce beam contamination, plasma formation, and impedance collapse. We have increased lithium current density a factor of 3 and lithium energy a factor of 5 through a combination of in-situ surface and substrate cleaning, impermeable substrate coatings, and field profile modifications.
\end{abstract}

\section{Parasitic Ion Production and Impedance Collapse in Ion Diodes}

Application of ion beams to Inertial Confinement Fusion (ICF) requires efficient production, extraction, transport, and focusing of an intense beam of an appropriate range ion ${ }^{1}$. Integrated target response calculations indicate that $600 \mathrm{~kJ}$ of $30 \mathrm{MV}$ lithium will allow high gain from an ion hohlraum ICF target. Toward this end we are studying the production of lithium beams from the LiF ion source on extraction ${ }^{2}$ applied-B ion diodes on the SABRE ${ }^{3}$ ( $\left.1 \mathrm{TW}, 5 \mathrm{MV}, 250 \mathrm{kA}\right)$ and PBFA- $\mathrm{II}^{4}(20 \mathrm{TW}, 10 \mathrm{MV}, 2$ $\mathrm{MA}$ ) accelerators. Important issues being addressed are diode impedance lifetime, ion current efficiency, ion beam lithium purity, uniformity, and divergence.

The lithium-fluoride $(\mathrm{LiF})$ lithium ion source is thought to operate by electron-assisted field desorption 5,6 . This source provides a pure lithium beam for $10-20 \mathrm{~ns}$, which is then replaced by contaminant ions for the remainder $(30-40 \mathrm{~ns})$ of the power pulse. Electric fields of $5-10 \mathrm{MV} / \mathrm{cm}$ are measured at the anode surface spectroscopically, via Stark shifts of the $6708 \AA 2 \mathrm{~s}-2 \mathrm{p}$ transition of neutral lithium ${ }^{5}$. These anode fields imply that the source is a field-threshold-emitter and that the lithium is not extracted from a surface plasma. After $10-20 \mathrm{~ns}$, visible and VUV emission spectroscopy show highly-ionized charge states of oxygen and carbon indicating plasma formation (electric field $=$ 0 ) in limited regions on the LiF surface and at the periphery of the LiF-coated emission area. The 6708 $\AA$ emission from the anode-cathode (A-K) gap results from non-resonant charge exchange with a $30 \mu \mathrm{m}$ thick, desorbed monolayer of contaminants near the anode. The spectroscopic evidence and measurements of time-resolved beam composition on both SABRE and PBFA-II indicate that the Li+ beam turns off while being replaced by a parasitic beam of hydrogen and carbon from local plasma regions forming on the anode. Appearance of the parasitic ion beam is accompanied by (although does not necessarily cause) a rapid impedance collapse, preventing efficient power coupling into lithium.

Our hypothesis is that the appearance of contaminant ions results from thermal desorption of neutrals from vacuum surface and bulk contamination, with subsequent avalanche ionization as the thickness and areal density of the expanding neutral layer reach critical values. Conventional vacuum surface cleaning techniques ${ }^{7-10}$ are therefore being applied to high power ion diodes on the SABRE ${ }^{11}$ and PBFA-II accelerators to control or eliminate surface and substrate contamination and their affects on diode operation. Initial experiments on diode cleaning using $R F$ discharges $7,8,10$, anode heating ${ }^{9}$, cryogenic cathode cooling 12,13, and impervious substrate coatings on the SABRE accelerator are discussed below. These techniques reduce anode and cathode plasmas and beam contamination, resulting in an increase in lithium ion beam enhancement, efficiency, energy, diode impedance, and a decrease in electron loss. The ultimate goal of this research is to determine the importance of surface 
and substrate contamination, and the level of cleaning required to operate a high power lithium diode with a negligible effect from contaminants. There are significant differences between ideal models of diode behavior and measurements ${ }^{5}$. These experiments are also the first step towards answering the question of how diodes behave without electrode contamination effects, and with reduced anode and cathode plasmas, effects not included in first-principal modeling.

SABRE experiments show the dominance of hydrocarbon ions and the associated anode plasma formation when the calculated anode substrate and/or source film temperature from electron bombardment reaches $200-400^{\circ} \mathrm{C}$. These temperatures are consistent with those required to generate anode plasmas in high power electron beam diodes ${ }^{14-17}$. IVORY 1-D electrostatic fluid simulations which self-consistently treat the thermal desorption, transport, ionization, and charge-exchange of neutral contaminants show good agreement with the parasitic load initiation on SABRE experiments ${ }^{18}$. These simulations show that thin, slow $(\approx 0.5 \mathrm{~cm} / \mu \mathrm{sec})$ thermally-desorbed neutral layers can quickly ionize, and possibly dominate the gap dynamics on the time-scale of the pulse (20 - $50 \mathrm{~ns})$. The critical condition for avalanche is that the neutral layer thickness be about twice the Larmor radius of secondary electrons $(\approx 100 \mu \mathrm{m})$ in the magnetic field near the anode (applied + diamagnetic $\approx 3-10 \mathrm{~T}$ ). The simulations ${ }^{18,19}$ also show that an ionizing neutral layer or partially-ionized plasma can be charge-exchange accelerated to velocities of $3-10 \mathrm{~cm} / \mu \mathrm{sec}$ within $15 \mathrm{~ns}$. The hypothesis of thermal desorption followed by avalanche breakdown implies that reducing the number of monolayers desorbed from the anode surface would reduce parasitic effects. This reduction can be accomplished by decreasing the anode temperature (through reduced electron loss, increased anode specific heat, or decreased initial anode temperature) or by reducing contaminant monolayers and increasing their binding energies (by in-situ surface and substrate cleaning). IVORY simulations confirm these solutions ${ }^{18}$ and predict that $\leq 2$ monolayers initial surface contamination yields a slow breakdown independent of anode temperature.

The production of fast neutrals can lead to loss of magnetic insulation as the ionizing neutral layer expands into the AK gap ${ }^{20}$. This leads to rapid enhancement of contaminant current, which clamps and shuts-off the lithium current, followed by impedance collapse. Desjarlais ${ }^{21}$ has also noted that the differences in voltage-current characteristics between the electric-field-threshold LiF and a spacecharge-limited parasitic ion source (which has a lower $V^{*}$ for the same Vcrit) demonstrate why LiF could be particularly susceptible to the turn-on of localized plasma regions which limit the lithium current even without plasma or neutral layer expansion. Theory and circuit simulations 20,21 of impedance collapse are consistent with the SABRE electrical data but the theory awaits strict experimental confirmation. There may be additional physical effects at work, and an integrated and more fully consistent picture of the impedance collapse is being developed.

\section{Cleaning Techniques}

Cleaning techniques have been used previously on applied-B diodes, motivated by the desire to improve lithium beam purity by reducing the hydrocarbon content of the anode plasmas ${ }^{22-26}$. The work described in this paper is unique in its motivation (mitigation of anode plasma formation), in the breadth, and intensity of cleaning techniques applied, in its application to a field-threshold ion source rather than a passive or active plasma source, and in the breadth and robustness of diagnostics. These experiments were also performed at higher diode power levels of $0.5-1 \mathrm{TW}$ at $5 \mathrm{MV}$ on a transit-timeisolated system, rather than on close-coupled systems at $0.05-0.25 \mathrm{TW}$, at $\leq 1 \mathrm{MeV}$.

Our principal surface cleaning technique was a capacitively coupled, 150 watt, $13.5 \mathrm{MHz}$ radiofrequency (RF) discharge in $5-10 \mathrm{mTorr}$ of Ar with 10 to $20 \% \mathrm{O}_{2}$ and pure Ar. The argon component of the discharge provides physical sputtering of surface contaminants. The oxygen component of the discharge provides chemical combustion, rapidly removing carbon and hydrogen from the surfaces. Discharge cleaning is one of the few ways to efficiently remove atomic carbon. The mixed gas discharge is immediately followed by a pure argon discharge which efficiently removes any non-reacted oxygen from the system via physical sputtering. The anode was the RF antenna and was electrically floated on a $0.5 \mathrm{~cm}$ nylon insulating spacer to maintain the discharge primarily in the $1-2 \mathrm{~cm} A-K$ gaps. RF lowers the threshold pressure compare to $\mathrm{DC}$ because all charged particles are not lost to the electrodes in a cycle. RF frequency was required to operate the discharge at sufficiently low pressures so that the impurities are not too collisional and can reach the vacuum pump. RF also prevents charging of the 


\section{DISCLAIMER}

This report was prepared as an account of work sponsored by an agency of the United States Government.' Neither the United States Government nor any agency thereof, nor any of their employees, makes any warranty, express or implied, or assumes any legal liability or responsibility for the accuracy, completeness, or usefulness of any information, apparatus, product, or process disclosed, or represents that its use would not infringe privately owned rights. Reference herein to any specific commercial product, process, or service by trade name, trademark, manufacturer, or otherwise does not necessarily constitute or imply its endorsement, recommendation, or favoring by the United States Government or any agency thereof. The views and opinions of authors expressed herein do not necessarily state or reflect those of the United States Government or any agency thereof. 


\section{DISCLAIMER}

Portions of this document may be illegible in electronic image products. Images are produced from the best available original document. 
dielectric LiF source films and allows cleaning of both the anode and cathode surfaces. Steady discharge operation was achieved with continuous gas feed and continuous pumping of the gas (and therefore sputtered impurities) by a turbo-molecular pump or cryopump.

The discharge pressure was optimized at $3-5$ mTorr by maximizing the impurity removal rate measured with a residual gas analyzer (RGA). The optimum pressure was a balance between increased sputtering rate of impurities at higher pressure and increased mobility of impurities out of the system at lower pressure. Pressures were set somewhat higher $(6-8 \mathrm{~m}$ Torr $)$ to prevent extinguishing the discharge as a result of small imbalances between gas feed and pumping speed for long, unsupervised cleaning cycles. RGA spectra with no cleaning, active cleaning with Ar only, and active cleaning with $\mathrm{Ar} / \mathrm{O}_{2}$ clearly indicate the highest impurity removal rates with $\mathrm{Ar} / \mathrm{O}_{2}$ discharges, particularly for $\mathrm{H}_{2} \mathrm{O}, \mathrm{CO}_{2}$, and $\mathrm{CO}$ impurities. The efficacy of various discharge cleaning protocols was measured with Xray Photoelectron Spectroscopy (XPS). They indicated that $15 \mathrm{~min}$ of an $\mathrm{Ar} / \mathrm{O}_{2}$ discharge was sufficient to reduce an initial $13-45$ monolayers of carbon impurities in the first $200 \AA$ of the LiF surface to $\leq 2-4$ monolayers.

A 40 - 120 second delay between cleaning and firing the accelerator was typical to pump out the discharge gas. Some experiments have used periods as short as 6 seconds. The SABRE accelerator base pressure was $1-8 \times 10^{-5}$ Torr, achieved with 9, eight-inch diameter cryo-pumps. About 10 monolayers/sec collide with the anode surface at a $10^{-5}$ Torr pressure. The anode surface recontaminates quickly if even a small fraction of these molecules stick. Discharge cleaning resulted in significant improvements despite the pumpdown delay and the base pressure, suggesting LiF has some resistance to recontamination. XPS measurements show a cleaned, room temperature LiF surface at $10^{-5}$ Torr adheres 10 carbon monolayers in 10 minutes. This should be compared to an initial $13-45$ layers following 8 - 24 hours vacuum exposure: We estimate 6 - 9 monolayers are present after a 2 minute delay on a room temperature surface. Anode recontamination during the pumpout delay was reduced by heating the anode $\left(200-450^{\circ} \mathrm{C}\right.$ for $6-30$ minutes) to reduce the surface sticking coefficient. The cathode was cooled with liquid nitrogen $\left(\approx-196^{\circ} \mathrm{C}\right)$ to lower the vacuum pressure in the anode-cathode gap $\left(\Delta \mathrm{p} \approx 2 \times 10^{-5}\right.$ Torr $)$ and reduce recontamination. The cryogenic cathode (cryo-K) also improves pumping of sputtered $\mathrm{H}_{2} \mathrm{O}, \mathrm{CO}_{2}$, and heaviest hydrocarbon contaminants during discharge cleaning. Cryogenic cooling has also been shown to delay breakdown associated with cathode plasma production in high voltage gaps ${ }^{12}$. RF discharges, anode heating, and cryo-K cooling may be an adequate protocol for cleaning of the LiF surface, except for the critical issue of surface re-contamination.

$\mathrm{LiF}$ film structure was examined with scanning electron microscopy (SEM). The structure is far from an ideal crystal, with long columns $(\approx 10-100 \mathrm{~nm}$ diameter) joined near the base at the substrate allowing significant effective surface area for trapping of contaminants, and large-scale regions $(\approx 5-50$ $\mu \mathrm{m}$ diameter) separated with cracks $(\approx 0.5-1.0 \mu \mathrm{m}$ wide). The long columns and large-scale cracks might allow transport of thermally-desorbed substrate contaminants to the diode region through the thin-film $(0.5-1.5 \mu \mathrm{m})$. Anode heating for short times (5 - 30 minutes) may provide enough cleaning through thermal desorption to eliminate contamination on the surface or in the bulk of the LiF film, but is inadequate to properly clean the stainless or titanium substrate below the LiF film in-situ ${ }^{9}$. These surfaces have hundreds of monolayers of surface oxides and weakly bound impurities 7,8 and constitute a practically infinite reservoir. Isolating the substrate impurities with a impermeable substrate coating under the LiF (discussed later) separates the surface from bulk cleaning issues and increases the efficacy of the above protocols.

\section{Extraction Ion Diode and Diagnostics}

The extraction applied-B ion diode inhibits electron conduction across the AK gap with a radial magnetic field $(2-3 \mathrm{~T})$ formed by four electromagnetic field coils. Two coils in the anode oppose the field produced by two coils in the cathode, creating the radial B-field. The four coils are separately energized to adjust the shape and magnitude of the insulating flux surfaces. Diode ion efficiency, and uniform beam extraction profiles are sensitive to the exact shape of the cathode electrode and magnetic flux surfaces ${ }^{2}$. The device forms an annular $70 \mathrm{~cm}^{2}$ extracted ion beam with a $5.5 \mathrm{~cm}$ mean emission radius and $2 \mathrm{~cm}$ width.

The accelerator voltage was obtained about $100 \mathrm{~cm}$ from the diode by measuring the arrival times and kinetic energies of protons accelerated across the Magnetically Insulated Transmission Line (MITL) 
gap with a magnetic spectrometer, and correcting for particle crossing time. This voltage history was corrected to the diode location using the MITL inductance. Diode, MITL, and ion beam currents were measured with B-dots. Ion beam currents were also measured with large aperture $(0.63-1.2 \mathrm{~cm}$ diameter) Faraday cups located $6 \mathrm{~cm}$ from the anode surface. The large aperture prevented aperture closure for at least $50 \mathrm{~ns}$. The cups were range-filtered with different thicknesses of mylar and aluminum to provide time-resolved species information. The cups sampled $0.3-2.5 \mathrm{~cm}^{2}(\approx 2 \%)$ of the beam area, normally with two cups for each filter to prevent non-uniformities from biasing the results.

A Thomson parabola (E parallel to B) mass spectrometer with CR-39 nuclear track recording film measured the time-integrated beam particle kinetic energy spectrum $(\mathrm{dN} / \mathrm{dE})$ as a function of the charge-to-mass ratio $(\mathrm{q} / \mathrm{m})$. This instrument collected the beam after Rutherford scattering at $7.5^{\circ}$ off a $2500 \AA$ thick free-standing gold foil located $11.5 \mathrm{~cm}$ from the anode. Rutherford scattering reduced the beam intensity to prevent aperture closure and assures measurement through minor beam optics variation. Analysis includes correction for Rutherford scattering, energy loss and stripping in the gold foil, and detection solid angle. The Thomson parabola sampled $<10^{-3} \mathrm{~cm}^{2}(<0.01 \%)$ of the beam and hence was highly sensitive to nonuniformity.

A lithium nuclear activation diagnostic 27 measured the lithium energy. This diagnostic was insensitive to contaminant species at our voltages $(<6 \mathrm{MV})$. Correction is made for the nuclear reaction yield as a function of beam kinetic energy and target angle. The lithium activation target sampled 9 $\mathrm{cm}^{2}(\approx 13 \%)$ of the beam area and was located $10 \mathrm{~cm}$ from the anode. Although there is beam loss in the $10-12 \mathrm{~cm}$ of vacuum transport before both the Thomson parabola and lithium activation target, these diagnostics are used to show a relative change in the quantity of lithium and impurities between different cleaning conditions.

Two time-resolved spectrometers collect visible ${ }^{5}$ and $V V^{28}$ emission spectra from anode, gap, and cathode chordal lines of sight. Stark shifts of the $6708 \AA 2 s-2 p$ transition of neutral lithium 5 measure the gap electric field with high-resolution visible spectra (to within $\pm 0.3 \AA$ ). Survey spectra over a wide range $(4000-7000 \AA)$ are used to investigate the affect of cleaning on plasma formation and content. The VUV is used strictly in a survey mode $(900-1600 \AA)$.

\section{Cleaning Results and Discussion}

Cleaning experiments were performed with two main ion diode B-field and electrode configurations. The initial diode ("config. $\mathrm{A}^{\prime}$ ) had all three cleaning techniques available (discharges, anode heating, cryo-K), but had limitations in diode efficiency and beam uniformity as a result of non-optimal magnetic field profile curvature. Cleaning resulted in significant lithium beam and impedance increase after peak ion power. We then improved the magnetic field profile and significantly increased beam uniformity, lithium current density and energy, and decreased electron loss without using cleaning. This new diode ("config. $B$ ") was used with the above cleaning techniques and impermeable source substrate coatings. No increase of lithium could be obtained with the new diode without coatings. The decreased electron loss achieved by modifying the B-field profile reduced the parasitic load significantly, making the required cleaning protocol for further improvement more difficult.

Fig. 1 compares electrical, particle, and spectroscopic diagnostics for cleaned (shot 1079) and uncleaned (shot 1092) shots with config. A. The open symbols throughout Fig. 1 had no cleaning for 3 previous shots. The solid symbols throughout Fig. 1 had 2.5 hours of discharge cleaning, 2 hours of cryo$\mathrm{K}$ cooling, 6 minutes of anode heating to $450^{\circ} \mathrm{C}$, with the anode hot at shot time, and a 2 minute pump out delay. These data show that an increase in lithium output and diode impedance after peak ion power, are correlated with a decrease in impurity ions and spectroscopic plasma emission.

Fig. 1a shows a comparison of voltage (circles), diode current (squares) and diode impedance (diamonds). These data show a dramatic effect of cleaning on impedance behavior for config. A, but the peak lithium power (p.l.p., marked with arrows) occurred at the same diode impedance for both cases $(50 \Omega)$. Fig. $1 \mathrm{~b}$ provides a comparison of diode impedances for averages of multiple cleaned and uncleaned shots. The error bars give upper and lower limits from the impedance histories in the average. The impedance history is about the same through p.l.p. Peak lithium power occurred at a diode impedance of $55 \pm 8 \Omega$ for the uncleaned case, and $50 \pm 7 \Omega$ for the cleaned case. The time of p.l.p. was delayed by about $5 \mathrm{~ns}$ with cleaning. The relative cleaned impedance was significantly higher 
only after p.l.p., perhaps on timescales where anode and cathode plasma reduction by cleaning had a significant impact on impedance. Comparing cathode current (Fig. 1a) and ion current output (Fig. 1c), we see that the overall lithium production efficiency with config. A was low $(8-16 \%)$ resulting from a poor field curvature. The diode impedance was therefore dominated by the electron loss in the diode, and this and beam turn-on delay could be additional reasons there was little effect on early diode impedance by cleaning.
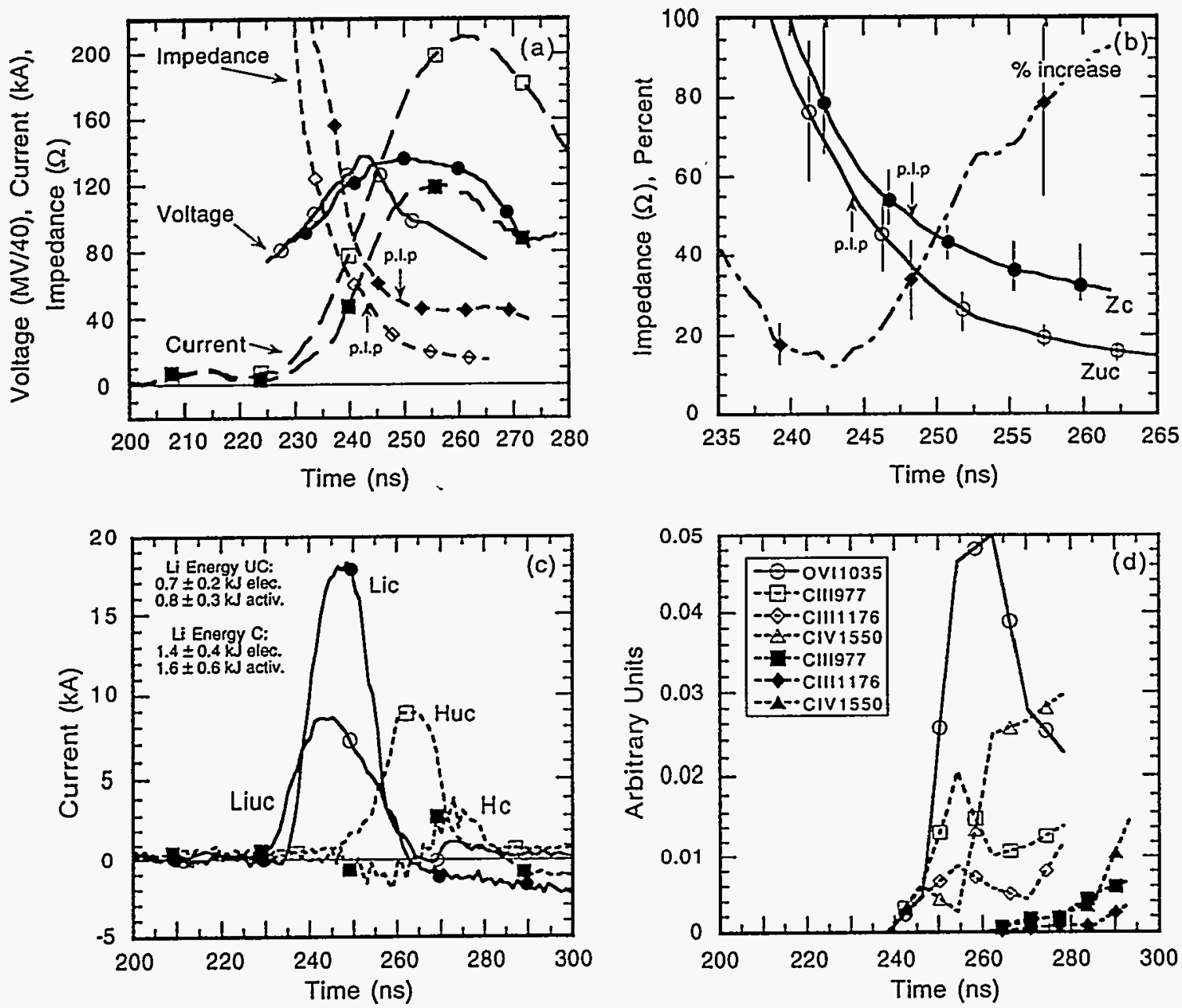

Figure 1. Part a, c, d, are comparisons for a specific pair of uncleaned (1092), and cleaned (1079) shots for config. A. "uc" or "uncleaned" and open symbols are used for uncleaned cases, "c" or "cleaned" and closed symbols for cleaned cases. Part b described below. A comparison of a) diode voltage (circles), current (squares), impedance (diamonds), b) average diode impedances for cleaned and uncleaned shots with config. A, c) a comparison of lithium (circles) and proton (squares) beam currents, and d) a comparison of anode VUV line intensities.

Fig. 1c compares lithium (circles) and proton (squares) ion current from large aperture filtered Faraday cups. The proton current was determined unambiguously from a cup with a $12.5 \mu \mathrm{m}$ thick aluminum filter, which registers only protons above $1 \mathrm{MeV}$. The $6 \mu \mathrm{m}$ cup registers protons, lithium above $3 \mathrm{MeV}$ and carbon above $6 \mathrm{MeV}$, (i.e. $\mathrm{C}^{2+}, \mathrm{C}^{3+}$ ). The lithium current was estimated from the difference between $6 \mu \mathrm{m}$ and $12.5 \mu \mathrm{m}$ aluminum-filtered cups, divided by the equilibrium lithium ion charge state. The Thomson parabola data (Fig. 2) shows that carbon above $6 \mathrm{MeV}$ was a small fraction $(12-18 \%)$ of the lithium above $3 \mathrm{MeV}$, even for an uncleaned shot, so the Faraday cup analysis gives an upper bound on lithium current. Electrical and nuclear diagnostics are in agreement on lithium beam energy which doubles with cleaning as noted on Fig. 1c. The proton current above $1 \mathrm{MeV}$ decreases by a factor of 2 , the charge by a factor of 10 , and the onset time was delayed by at least 30 ns between the 
uncleaned and cleaned shots. The increase in lithium appears to be correlated directly to an elimination of early proton current (up to $1 \mathrm{kA}$ at $245 \mathrm{~ns}$ ). Unfiltered cups (not shown) imply that there was also carbon in the beam early, consistent with the Thomson parabola data in Fig. 2. Cleaning does not appear to be as effective in removing the carbon. Space-charge limited carbon emission sites can still effectively complete for diode power with the field-threshold lithium emission sites. The decrease in cathode current (Fig. 1a), and simultaneous increase in lithium current (Fig. 1c) implies a reduction in electron loss with cleaning.

Fig. 1d compares VUV spectroscopic observations of $a \approx 1 \mathrm{~mm}$ region including the anode surface. Cleaning delayed VUV onset by $25 \mathrm{~ns}$ and resulted in a slower rate of rise of the CIII and CIV lines at 977,1176 , and $1550 \AA$. Oxygen lines were reduced by more than a factor of 10 ; OVI at $1035 \AA$ shown in Fig. 1d for the uncleaned case. Shots without heating do not show as strong a reduction in $O$ lines. The plasma emission for the uncleaned shot begins near peak lithium output ( $\approx 245 \mathrm{~ns}$, see Fig. 1c). Uncleaned and cleaned shots show a simultaneous measurement of high $(\approx 8 \mathrm{MV} / \mathrm{cm})$ electric fields ${ }^{5}$ near the anode surface during periods with intense plasma light, suggesting non-uniform plasma production and different emission locations and source physics for the lithium and contaminant beams. The increase of lithium (Fig. 1c) is more strongly correlated with the decrease or delay in anode plasma production (Fig. 1d), and in beam contamination (Fig. 1c), than to a decrease in the rate of impedance collapse (Fig. 1a, b).
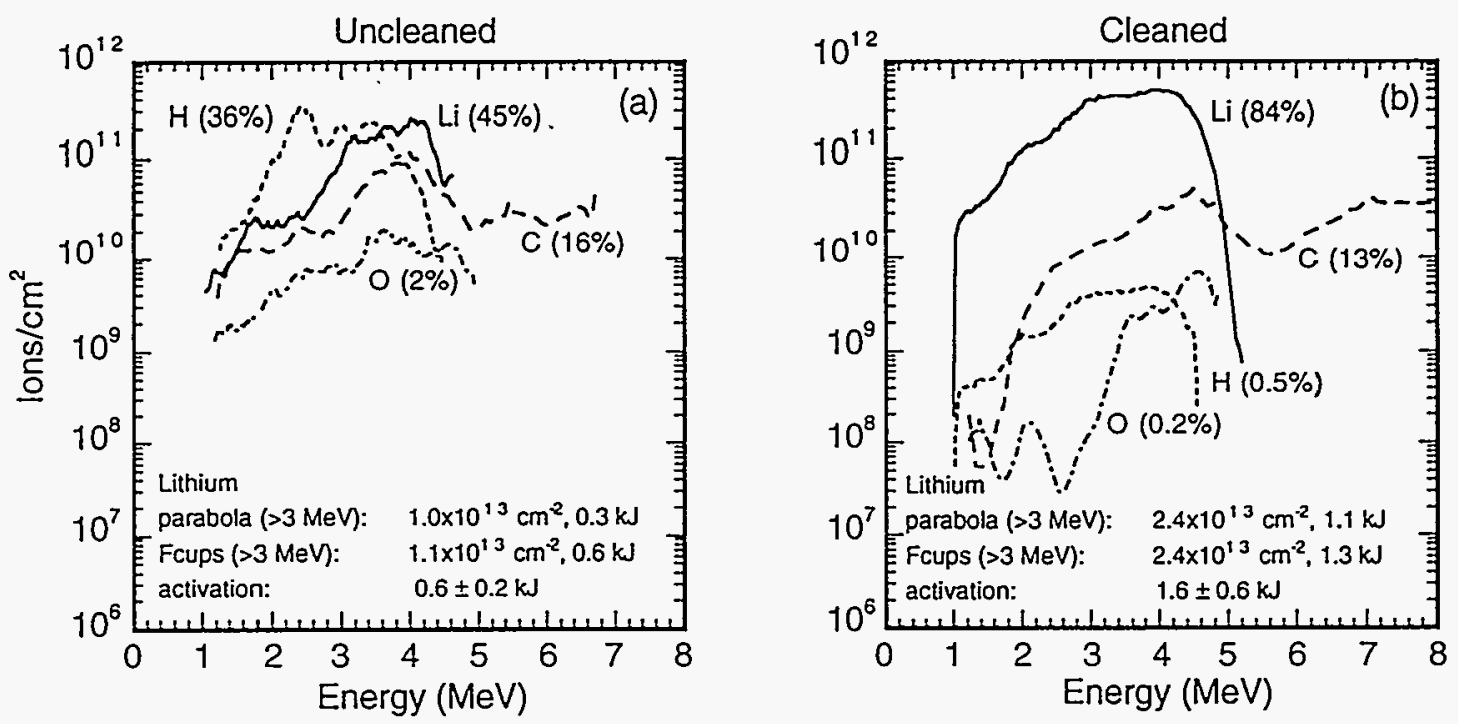

Figure 2. A comparison of the Li, H, C, O time-integrated particle energy distributions from unfolded Rutherford scattering Thomson parabola for a) uncleaned shot 1101 and b) cleaned shot 1079 , config. A.

Fig. 2 shows a spectral unfold from the Rutherford scattering Thomson parabola mass spectrometer comparing an uncleaned shot in part 2a (1101, the same case as 1092 in Fig. 1) with a cleaned shot in 2b (1079, the same shot shown in Fig. 1), for config. A. The lithium, hydrogen, carbon, and oxygen timeintegrated particle energy spectra are plotted. Fluorine was about the same fraction as the oxygen in general. In this comparison, cleaning raised the lithium beam fraction from $45 \%$ to $84 \%$, corresponding to an increase of 2.5 in lithium production, and reduced protons from $36 \%$ of the beam to less than $1 \%$. Note in Fig. 2a that the proton beam dominated the lithium beam at lower energies (below $3.5 \mathrm{MeV}$ ) a trend consistent with Faraday cup observations described in Fig. 1c. Proton fluence was an order of magnitude lower than lithium for energies above $3.5 \mathrm{MeV}$, also consistent with the Faraday cups in Fig. 1c. The presence of carbon above $5 \mathrm{MeV}$ implies that multiply ionized charge states $\left(\mathrm{C}^{2+}, \mathrm{C}^{3+}\right)$ were accelerated in the gap.

As noted on Fig. 2, Thomson parabola, filtered Faraday cups, and lithium nuclear activation alf show lithium current increases with cleaning, and are in reasonable agreement. The agreement with the Thomson parabola is possibly somewhat fortuitous since it is quite sensitive to beam non-uniformity 
because of small sampling area, and to losses of the beam over an additional $6 \mathrm{~cm}$ of vacuum transport. Table I summarizes time-integrated Thomson parabola data averaged over several cleaned and uncleaned shots for the config. A to account for the sampling limitations. The visible and VUV spectroscopic data imply that plasma formation is non-uniform, hence averaging the beam composition data over multiple shots is essential, particularly for contaminant ions. The carbon beam fraction was not affected by the cleaning techniques used (RF discharges, cryo-K, anode heating to $450^{\circ} \mathrm{C}$ for 6 minutes just before the shot). The large reduction in protons and no reduction in carbon suggests that the carbon is in a form or located in the LiF bulk or substrate such that these cleaning techniques have not been effective. Fluorine and oxygen were negligible compared to carbon and hydrogen and were unaffected by cleaning to within the uncertainties of the diagnostic.

Table I. Summary of Unfolded Time-Integrated Beam Composition from Rutherford Scattering Thomson Parabola - Config. A

\begin{tabular}{c||cccc}
\multicolumn{1}{c||}{ Case } & Lithium & Hydrogen & Carbon & Fluorine + Oxygen \\
\hline \hline $\begin{array}{c}\text { No Cleaning } \\
\text { (average of } 6 \text { shots) }\end{array}$ & $44 \pm 18$ & $35 \pm 20$ & $17 \pm 7$ & $4 \pm 4$ \\
$\begin{array}{c}\text { Cleaning } \\
\text { (average of } 7 \text { shots) }\end{array}$ & $69 \pm 14$ & $10 \pm 9$ & $19 \pm 10$ & $3 \pm 1$
\end{tabular}

Fig. 3 plots the lithium nuclear activation signal in cts/min (raw signal) versus the strength of the insulating magnetic field Vcrit (MV) for a variety of experimental conditions. The raw activation signal is taken as an indication of total lithium energy, good to a first approximation since the voltages are similar. The lithium energy from a full activation analysis and from the electrical diagnostics is noted for a few cases in proof. There is some disagreement between electrical and activation energy at the higher levels that requires further study. Open symbols are cases with no cleaning, and solid symbols cases with some form of cleaning. Symbols with error bars are the averages of 2 to 4 shots; otherwise the symbols represent a single shot.

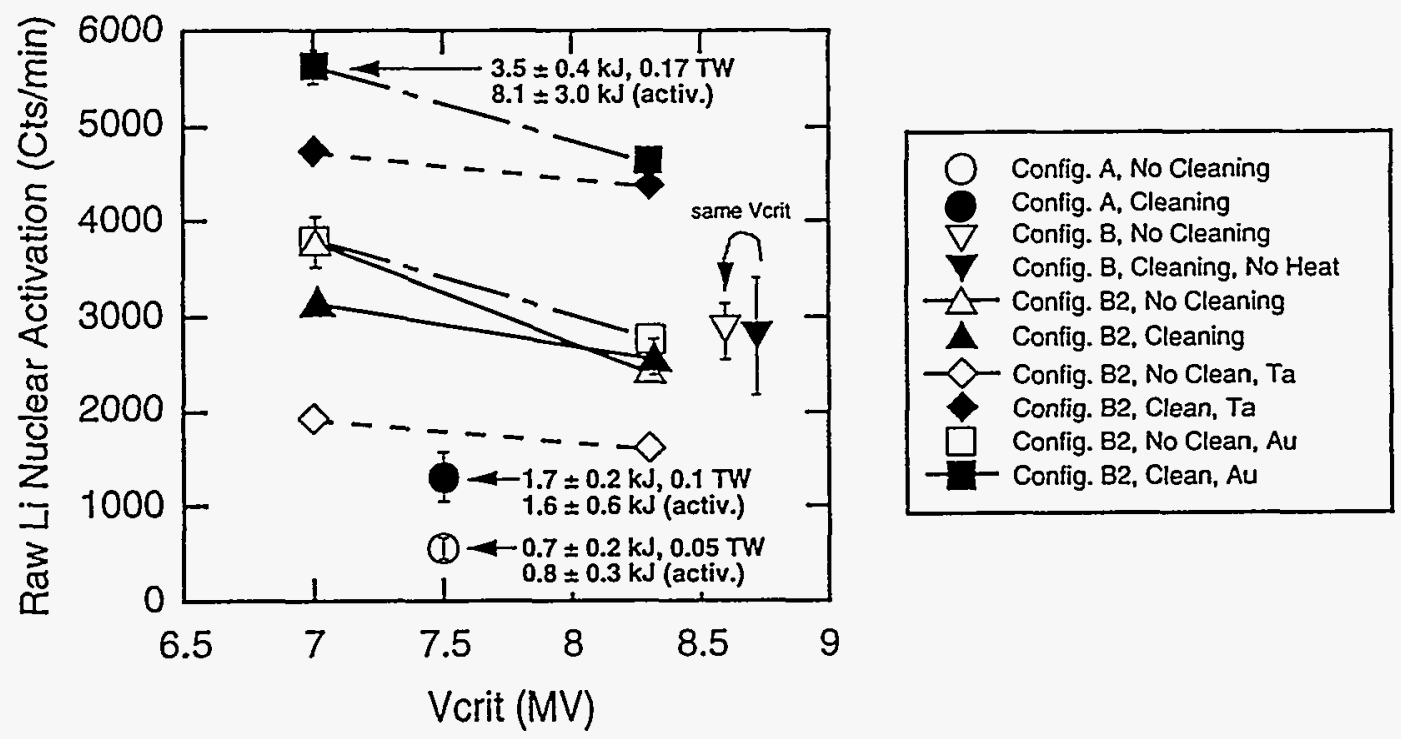

Figure 3. A summary of the raw lithium nuclear activation signal for various SABRE diode configurations with and without cleaning. Lines are shown to connect similar configurations.

Early promising results of cleaning with config. A (open and solid circles in Fig. 3, example results discussed above in Figs. 1,2) were not immediately obtained with config. B with the same cleaning 
protocols (open and solid triangles, open and solid inverted triangles, Fig. 3). Heating and discharge cleaning were tested on PBFA-II with similar observations. Our hypothesis is that efficient field profiles with config. B and on PBFA-II, lowered electron loss and therefore thermal desorption of contaminants, making the sources more resistant to the remaining level of surface contamination. If so, config. $B$ requires improved cleaning to give a further improvement of performance. There are two subsequent hypotheses for the requirement of improved cleaning: 1) neutrals come from re-contamination of the anode surface during the discharge pump out delay before firing; and 2) neutrals are desorbed from the metal substrate below the permeable LiF source films. Preparation is underway for reduced base pressures $\left(<5 \times 10^{-6}\right.$ Torr) and short pump out delays ( $<10$ seconds) for a test of hypothesis 1 . Thin films of $\mathrm{Ta}$ and $\mathrm{Au}(\approx 0.5 \mu \mathrm{m}$ thick) below the $\mathrm{LiF}$ ion source film have been used to evaluate hypothesis 2 , discussed below. Possible effects of thin-film substrate coatings on the parasitic source behavior include providing an impermeable coating to seal the substrate material, and changing the substrate specific heat to demonstrate unambiguously if the mechanism for parasitic load onset is thermal. We find that these films dramatically affect the lithium output and parasitic load behavior.

The addition of Ta substrate coatings, without cleaning (open diamonds, Fig. 3), immediately lowers the lithium output by about a factor of 1.5 to 2 compared to the null cases without $\mathrm{Ta}$, with or without cleaning (open and solid triangles, Fig. 3). The specific heat of Ta is a factor of $\approx 3.5$ lower than for $\mathrm{Ti}$ or stainless anodes, thus the anode temperature rise, thermal desorption and neutral avalanche occur faster. The reduction in lithium is correlated with an observed early onset of parasitic load (early onset of large proton filtered Faraday cup signals) resulting from increased heating and neutral desorption. Cleaning with Ta coatings (solid diamonds, Fig. 3 ) results in a significant increase ( $x$ 2.5) above the uncleaned case. The Ta coatings are apparently easier to clean in-situ with shortperiod heating ( $<30$ minutes) than the original stainless or Ti substrate material and seem to provide an impermeable seal for the substrate.

The Au substrate coatings (open squares, Fig. 3) do not result in a lower lithium output despite an $\approx$ 3.9 times lower specific heat, perhaps because Au films are more contamination resistant than $\mathrm{Ta}$ and have fewer adsorbed monolayers at shot time. Cleaning with Au coatings (solid squares, Fig. 3 ) results in the largest lithium output of any configuration yet. Anode VUV spectroscopy does not show carbon impurity line emission for the case of Au substrate with cleaning. The appearance of carbon lines is delayed longer than the instrument recording time. VUV carbon emission lines have previously always been observed without cleaning, as well as with all other cleaning protocols. The cleaning protocol for all of the substrate coating experiments (Config. B2 in Fig. 3 legend) used 2-5 hours of RF discharges and cryogenic cathode cooling, simultaneously with about $15-20$ minutes anode heating at $200^{\circ} \mathrm{C}$, and $6-10$ minutes at $400^{\circ} \mathrm{C}$ just before shooting, with a $40 \mathrm{sec}$ pumpout delay. The observations with $\mathrm{Ta}$ and $\mathrm{Au}$ coatings indicate that the substrate material impacts source performance and that thermal desorption is likely the dominant contributor to parasitic load initiation.

\section{Summary}

These results generally show the importance of thermal desorption of neutrals from the anode, leading to formation of anode plasma on the $\mathrm{LiF}$, and the participation of anode and cathode plasmas in impedance collapse in ion diodes. We also showed that: 1) surface cleaning could mitigate part of the parasitic load, and increase lithium production and diode impedance (after peak lithium power); 2) reduced surface re-contamination is probably necessary to keep the anode surface clean until shot time; 3) the LiF films were permeable, allowing substrate contaminants to enter the diode; 4) the cleaning techniques we were using did not affect contaminants from the $\mathrm{Ti}$ or stainless substrates; 5) and the diode performance depends on the type of substrate material. These data with different substrates are the first demonstration that substrate as well as surface contaminants participate in the diode operation with the LiF source and that the mechanism behind parasitic load initiation is likely to be thermal since the specific heat of the substrate matters. Comparison of the results with configs. A and $B$ suggest that a more dramatic improvement of lithium intensity with cleaning (of $L i F$ ) is obtained when: the electron loss is higher (seen on config. A), when the specific heat is lower (seen with Ta coatings), and when the contaminant level is initially larger (Ta versus Au coatings). As electron loss is decreased, more thorough cleaning is required to impact performance. These observations are consistent with scaling conclusions drawn from IVORY models of neutral layer desorption and avalanche ${ }^{18}$. 
Overall we have seen a factor of 3 increase in lithium current density and factor of 5 increase in lithium energy through a combination of surface and substrate cleaning, impermeable substrate coatings and field profile modifications as we progressed from config. A without cleaning (open circles, Fig. 3) to config. B with full cleaning and Au substrates (solid squares, Fig. 3). Cleaning with config. A gave about a factor of two increase in lithium energy and power compared to the uncleaned case. Cleaning and coatings with config. B give about a factor of $40-60 \%$ increase in lithium current density and $50-100 \%$ increase in lithium energy compared to the uncleaned case. These performance improvements have resulted from reduction or delay in production of anode and cathode plasmas achieved with control of electron loss, anode heating, and surface and substrate contaminants. Cleaning techniques show considerable promise for control of anode and cathode plasmas in short-pulse, high-energy-densityexperiments.

Further development of advanced cleaning techniques for control of surface and substrate contaminants is proceeding. Experiments with lower base pressures, shorter pumpout delays, and impermeable, high-specific-heat films are planned, in conjunction with higher power discharges, anode heating and cathode cooling. Possible high specific heat films are boron or aluminum $\approx \approx$ factor of 2 higher than stainless or titanium), and annealed LiF films ( $\approx$ factor of 3 higher). According to IVORY simulations and some experimental evidence, $\leq 2$ monolayers is required to keep the neutral avalanche slow, independent of anode temperature. This is our target level for cleaning. Cleaning techniques are also essential for the development of non-protonic active sources. Non-protonic active sources are particularly susceptible to desorption of surface impurities, since either ohmic or laser energy is delivered to the anode surface prior to arrival of the power at the diode gap. This energy can desorb physically- or possibly chemically-adsorbed surface and bulk impurities at lower temperatures than those at which lithium is released from the source films $11,25,26,29$.

The authors would like to acknowledge C. L. Olson, T. A. Mehlhorn, and J. E. Maenchen, for support and encouragement, C. L. Ruiz for lithium activation calibrations, and W. A. Stygar for development of the VIDA nuclear track counting system. The technical support of D. Nielson, D. Tanner, J. Potter, B. Lewis, D. Pariza, W. Stearns, P. Reyes, and A. Schmidlapp is gratefully acknowledged. This work is supported by the U. S. DOE under contract no. DE-AC04-94AL85000.

\section{References}

1. J. P. VanDevender, and H. Bluhm, Chapter 18, in Nuclear Fusion by Inertial Confinement, eds. G. Velarde, Y. Ronene, J. M. Martinez-Val (CRC Press, Ann Arbor, 1993).

2. D. L. Hanson, et al., Proc. 10th Inter. Conf. on High Power Particle Beams, NTISPB95-144317, 418(1994).

3. M. E. Cuneo, et al., Proc. 9th Inter. Pulsed Power Conf., IEEE Cat. No. 93CH3350-6, 423(1993).

4. T. A. Mehlhorn et al., Proc. 10th Inter. Conf. on High Power Particle Beams, NTISPB95-144317, 53(1994).

5. J. E. Bailey, et al., Phys. Rev. Lett., 74, 1771(1995)

6. T. A. Green, prvt. comm., 1992; also R. W. Stinnett, et al., Proc. 9th Conf. High Power Particle Beams, NTISPB92206168, Vol. II, 788(1992).

7. H. F. Dylla, J. Vac. Sci. Tech. A, 1276(1988).

8. H. F. Dylla, J. of Nuc. Mater., 93\&94, 61(1980).

9. M. H. Achard, R. Calder, and A. Mathewson, Vacuum, 29, 53(1978).

10. H. C. Hseuh, T. S. Chou, and C. A. Christianson, J. Vac. Sci. Tech. A, 3, 518(1985).

11. M. E. Cuneo et al., BAPS 36, 2423(1991); also IEEE Conf. Plasma Science, IEEE Cat. No. 91CH3037-9, 94(1991).

12. G. A. Mesyats, D. I. Proskurovsky, Pulsed Electrical Discharge in Vacutum, (Springer-Verlag, 1988).

13. D. L. Hanson, et al., J. Appl. Pjys., 70, 2926(1991).

14. D. W. Swain, et al., J. Appl. Phys., 48, 1085(1977).

15. A. E. Blaugrund, G. Cooperstein, S. A. Goldstein, Physics Fluids, 20, 1185(1977).

16. T. W. L. Sanford et al., J. Appl. Phys., 66, No. 1, 10(1989).

17. M. E. Cuneo, Ph. D. Thesis, Univ. of Michigan, 1989, Univ. Microfilms Inter., 89-20519; also M. E. Cuneo, et al., IEEE Trans. Plasma Sci., PS-15, 375(1987).

18. D. R. Welch, MRC Report ABQ-N-553(1994); also D. R. Welch, M. E. Cuneo, these proceedings.

19. T. D. Pointon, J. Appl. Phys., 66, 2879(1989); also D. S. Prono, et al., J. Appl. Phys., 52, 3004(1981).

20. M. P. Desjarlais, Physics Fluids B, 1, 1709(1989).

21. M. P. Desjarlais, Bull. Amer. Phys. Soc., 37, 1534(1992).

22. K. W. Bieg, et al., J. Vac. Sci. Tech. A, 3, 1234 (1985), also J. Vac. Sci. Tech. A, 4, 772(1986).

23. E. J. T. Burns, et al., J. Appl. Phys., 63, 11(1988).

24. J. J. Moschella, et al., J. Appl. Phys., 70, 3418(1991).

25. C. K. Struckmann, and B. R. Kusse, J. Appl. Phys., 74, 3658(1993).

26. G. C. Tisone, et al., Proc. 9th Conf. High Power Particle Beams, NTISPB92-206068, Vol. II, 800(1992).

27. R. J. Leeper, et al., Nucl. Instr. Meth. in Phys. Res., B24/25, 695(1987).

28. A. B. Filuk, et al, Proc. 10th Inter. Conf. High Power Particle Beams, NTISPB95-144317, 414(1994).

29. A. B. Filuk, et al., Proc. 9th Conf. High Power Particle Beams, NTISPB92-206068, Vol. II, 794(1992). 